\title{
The magnetic nature of wide EUV filament channels and their role in the mass loading of CMEs
}

\author{
G. Aulanier ${ }^{1}$ and B. Schmieder ${ }^{1,2}$ \\ 1 Observatoire de Paris, LESIA, 92195 Meudon Cedex, France \\ e-mail: brigitte.schmieder@obspm.fr \\ 2 University of Oslo, ITA, PO Box 1029, Blindern, 0315 Oslo, Norway
}

Received 26 November 2001 / Accepted 23 January 2002

\begin{abstract}
Previous works have shown that dark and wide EUV filament channels observed at $\lambda<912 \AA$ are due to absorption of EUV lines in cool plasma condensations that are not observed in $\mathrm{H} \alpha$. We extend this interpretation and we address the issue of the possible injection of their mass into CMEs, through the magneto-hydrostatic modeling in 3D of one filament observed both in $\mathrm{H} \alpha$ and in EUV. The model parameters are fixed so as to match the $\mathrm{H} \alpha$ observations only. Further comparison of the model with the EUV observations reveal the magnetic nature of the absorbing plasma condensations. They are formed in magnetic dips, as for the filament itself. Opacity ratios and the hydrostatic condition imply that the dips must be filled by cool material up to $1700 \mathrm{~km}$, which increases the filament mass by $50 \%$ as compared to $\mathrm{H} \alpha$ estimations. Far from the filament, the absorbing condensations are located below $4 \mathrm{Mm}$ in altitude above the photosphere, on the edge of weak photospheric parasitic polarities, within the lower parts of long field lines overlaying the filament. By physical analogy with $\mathrm{H} \alpha$ filament feet, we redefined these extended regions as EUV feet. The broadening of the EUV filament channel is dominated by EUV feet, while the larger filling of dips plays a non-negligible but minor role. Further implications from this work are discussed, on the visibility and the geometry of the condensations, on the existence of EUV filament channels in the absence of filaments, on the loading of cool material into filament feet through bald patch reconnection and on the complex geometry of the upper prominence-corona transition region. The magnetic topology implies that during the filament eruption, the mass of its wide EUV feet will not contribute to the CME, whereas the extra mass provided by the large filling of dips in the filament flux tube will be loaded into the CME.
\end{abstract}

Key words. Sun: chromosphere - filaments - magnetic fields - UV radiation - solar-terrestrial relations

\section{Introduction}

Solar filaments/prominences in their quiescent phase are the largest and best observed coronal structures in which dense plasma is confined in highly stressed magnetic fields. These fields contain a large amount of free magnetic energy, which can eruptively be released through several processes (see the reviews of Forbes 2000; Klimchuk 2001; Low 2001). This naturally explains why some CMEs are associated with filament/prominence eruptions (see e.g. van Driel-Gesztelyi et al. 1998; Schmieder et al. 2000; Delannée 2000; Marqué et al. 2002).

The total mass embedded in a CME has been estimated from coronograph observations in the range of $10^{15-16} \mathrm{~g}$ (Gosling et al. 1974; Howard et al. 1985; Hundhausen 1988). Typical prominence masses are difficult to measure due to uncertainties in the estimation of ionization degree and the filling factor (see the reviews

Send offprint requests to: G. Aulanier, e-mail: guillaume.aulanier@obspm.fr of Schmieder 1989; Vial 1990). Considering a typical Ho prominence as a thin volume whose thickness, length and height are respectively 5, 100 and $40 \mathrm{Mm}$ and taking a mean proton density of the order of $10^{10-11} \mathrm{~cm}^{-3}$, then typical prominence masses range in $3 \times 10^{14-15} \mathrm{~g}$. So, erupting filaments may account for a non negligible fraction of the total mass of their related CMEs.

Recent findings on wide EUV filament channels (detailed below) suggest that filaments may contain much more mass than what is shown by $\mathrm{H} \alpha$ observations. Moreover, about $40-60 \%$ of all CMEs are associated to filament/prominence eruptions (Delannée et al. 2000; Subramanian \& Dere 2001). So independantly of coronographic measurements, the fraction of the mass from a wide EUV filament channel that can be loaded into a CME when its related filament erupts needs to be estimated. This issue is important for solar-terrestrial relations because the kinetic energy of a propagating CME is an important parameter to study its interaction with the magnetosphere. 
In EUV, the typically observed structure of a filament is a wide region located along the $\mathrm{H} \alpha$ filament axis, which corresponds to an intensity depletion. This region is usually referred to as the "EUV filament channel" (hereafter EFC). These structures have often been reported from Skylab and OSO-4 and -6 observations (Orrall \& Schmall 1979, 1979), and are now frequently observed with SoHO instruments such as SUMER, CDS (Kucera et al. 1999; Chiuderi Drago et al. 1998, 2001; Heinzel et al. 2001; Schmieder et al. 2002) and EIT (Wang 2001, see also Fig. 1), as well as with the TRACE satellite (Chae 2000). The width of an EFC can vary from 10 to $50 \mathrm{Mm}$ along its axis, which is in average 5 times wider than its related $\mathrm{H} \alpha$ filament body.

Chiuderi Drago et al. $(1998,2001)$ have reported that for emission lines of a given ion, EFCs are not observed for wavelengths larger than $912 \AA$, whereas they are clearly visible for shorter wavelengths. Therefore it has been proposed that EFCs are due to the absorption of the background EUV radiation by the Lyman continuum of hydrogen, in suspended cool plasma condensations in which neutral hydrogen is present. Heinzel et al. (2001) and Schmieder et al. (2002) have shown that such condensations are visible in EUV on each side of $\mathrm{H} \alpha$ filaments. The lack of $\mathrm{H} \alpha$ absorbing material in EFCs has been explained by opacity ratios: with NLTE models, Heinzel et al. (2001) have calculated that for typical prominence temperatures, the opacity for $\mathrm{H} \alpha$ is typically $50-100$ times lower than the opacity at the head of the Lyman continuum of hydrogen.

Therefore EUV observations of filaments and their surroundings reveal a much larger distribution of mass than for their related $\mathrm{H} \alpha$ filaments. The aim of the present study is to explain (i) how cool plasma condensations (which absorb EUV radiation) can be formed aside of a filament body and away from its feet (thus being able to form a wide EFC), to identify (ii) what is the magnetic field in and around these condensations, (iii) what is their altitude in the solar atmosphere, (iv) what is their relation with the filament magnetic fields, and to estimate $(v)$ if they can contribute to the CME mass when the related filament eventually erupts.

To address these issues, we perform a linear magnetohydrostatic (LMHS) model of one intermediate filament observed on May 5, 2000 at E17 S21, during a coordinated campaign between THEMIS and SoHO, conducted at the MEDOC operation center. We used a line of sight magnetogram as boundary conditions, and we compared the calculated magnetic features with the EUV observations.

The paper is organized as follows: in Sect. 2, we list the multi-wavelength observations of the studied filament and we describe specific features. In Sect. 3, we explain the computational method to calculate linear magnetohydrostatic configurations, and we construct a grid of 35 models for various values of the free parameters. In Sect. 4 , we select one of the models so as to match the $\mathrm{H} \alpha$ data independantly of the EUV data, we give physical interpretations of the $\mathrm{H} \alpha$ filament and we describe the resulting magnetic topology of the filament flux tube. In Sect. 5, we estimate the plasma filling of magnetic dips and we show that calculated low-lying magnetic dips match the complex shape of the observed EFC, which provides direct answers to the issues $(i-i v)$ addressed above. In Sect. 6, we compare our modeled topology with other prominence models and with $\mathrm{H} \alpha$ observations of filaments. We also discuss further implications derived from the model on the injection, the distribution and the visibility of mass in EFCs, on the possible existence of EFCs in the absence of filaments, and finally on the fragmented nature of the upper prominence corona transition region. In Sect. 7 we address the issue $(v)$ and we show that the model predicts that the wide extensions of EFCs will not contribute to the mass loading of CMEs so that only the mass contained in the filament flux tube will be ejected. A summary of the results is given in Sect. 8 .

\section{Multi- $\lambda$ observations of the filament}

\subsection{Available dataset}

This paper is focused on the analysis of one filament observed on May 5, 2000 at E17 S21, during a MEDOC campaign with the MSDP of the franco-italian telescope THEMIS and with SUMER and CDS onboard the SoHO spacecraft. The full description of the data acquisition and of the dataset is provided in Heinzel et al. (2001) and in Schmieder et al. (2002).

In the present paper, we use two intensity images from this campaign: one of the MSDP in $\mathrm{H} \alpha$, obtained at 7:52 UT, and one of CDS in Ov at $629.73 \AA$, obtained at 8:12 UT for which 51 min were necessary to do the raster. We choose this wavelength because it displayed mostly the same dark features than in the 5 other recorded lines (Si XII [520.60 $]$ ], Ca x [557.77 $\mathrm{A}$ ], Ne VI

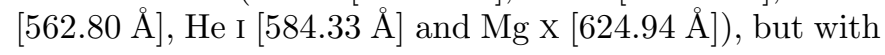
a better contrast. For the modeling work we also consider one SoHO/MDI line of sight magnetogram, obtained at 8:00 UT (shown in Fig. 1).

\subsection{Co-alignment of the observations}

For the aim of the present study, a good co-alignment between the data is crucial, especially for the comparison of models with the observations. But since the MSDP and CDS data are not full disc, we co-align the observations in several steps.

First we overlay the dark filament observed with THEMIS with a full disc Meudon $\mathrm{H} \alpha$ spectroheliogram observed at 10:18 UT (a partial view is shown in Fig. 1). Then we overlay the Meudon full disc image (after translating it to account for solar rotation) with the MDI data. In order to minimize the effects of the evolution of the structures between 8:00 and 10:18 UT, we fine-tune this overlay so that the bright $\mathrm{H} \alpha$ plages match the strong magnetic fields as well as possible. In a third step, the CDS image is overlaid with these data by matching the EUV network brightenings with the strongest magnetic 

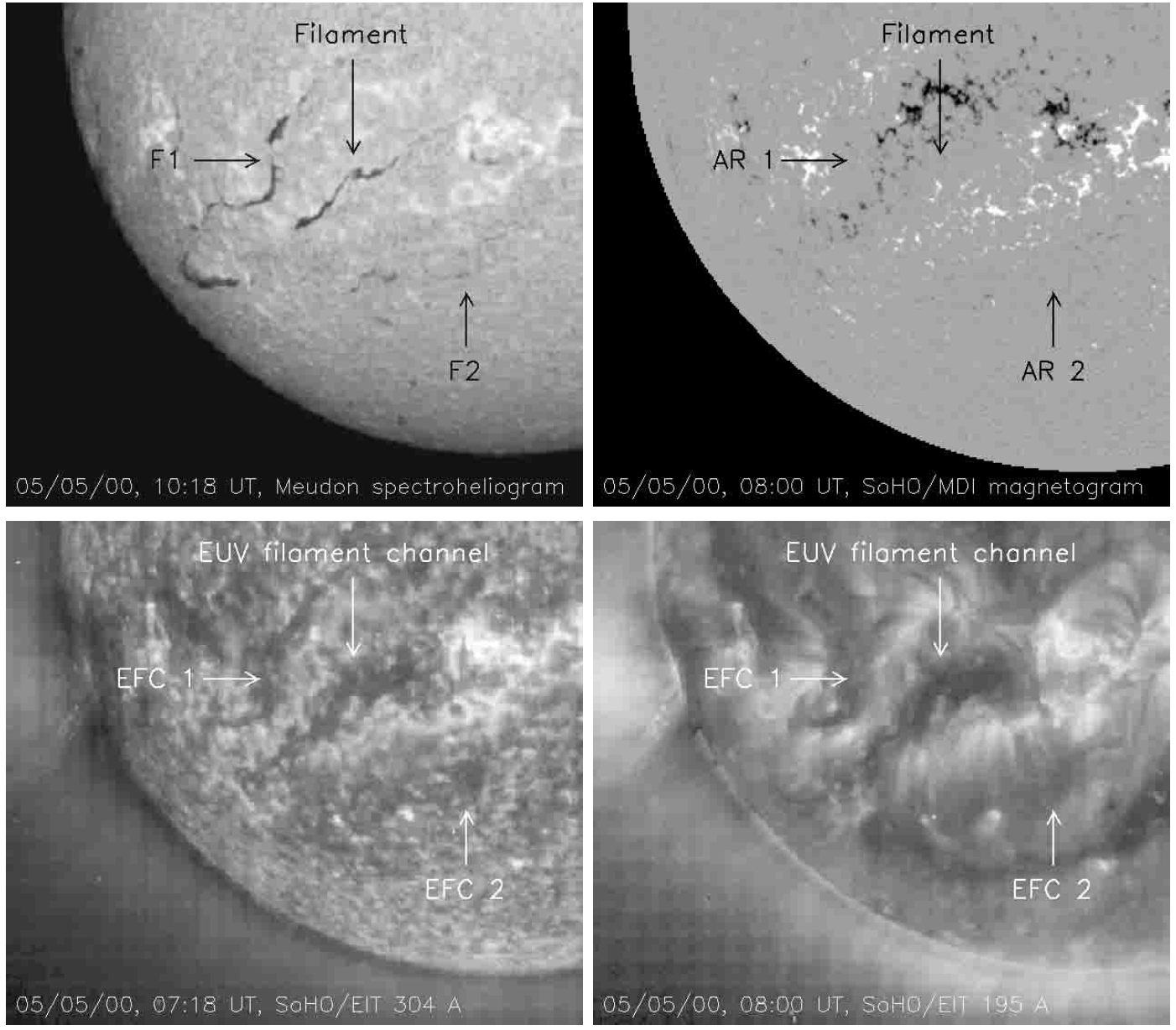

Fig. 1. Partial view of full disc data from the Meudon spectroheliograph in $\mathrm{H} \alpha$ and from SoHO/MDI and EIT. The studied filament is located at E17 S21, between two decaying active regions (AR1 and AR2) which both also contain an H $\alpha$ filament (F1 and F2). The three filaments are embedded in their own EUV filament channel, as indicated by arrows in the lower panels.

fields, using an iterative tracking of pre-selected features such as network brightenings.

Note that during all these steps, we do not modify the magnetogram, which otherwise could reduce the reliability of the modeling (described in Sect. 3). The resulting coalignment of the THEMIS, CDS and MDI data is shown in Fig. 2. As pointed out by Heinzel et al. (2001), the filament in $\mathrm{H} \alpha$ is thinner than the EUV filament channel (EFC) by a factor 5 in average.

\subsection{Noticeable features}

Figure 1 shows that the studied filament is located between two decaying active regions (AR1 and AR2), between faculae of opposite polarities, which is typical of $75 \%$ of filaments (d'Azambuja \& d'Azambuja 1948; Tang 1987). Two other intermediate filaments F1 and F2 are present, on the neutral line of AR1 and AR2 respectively. On that day, the filament F2 was not yet well formed, but both the EUV filament channels for F1 and F2 are clearly visible.

Figure 2 (top) shows that the $\mathrm{H} \alpha$ filament has an elbow located at the edge of a strong magnetic concentration of positive polarity. This leads to a curved "S-shape" for the filament, which may be interpreted as a sigmoid. We discuss in Sect. 4.2 the validity of this interpretation. The filament feet clearly have a left-bearing chirality. Since the filament is in the southern hemisphere, the feet follow the chirality rules found by Martin et al. (1994). At first look, the feet can hardly be associated with strong parasitic polarities, but a careful examination of the coaligned data permits us to identify several weak parasitic polarities $\left(B_{\|} \simeq 3-10 \mathrm{G}\right)$ that are well associated with the feet (see Sect. 4.3), in accordance with Martin (1990), Aulanier et al. (1999) and Wang (2001). Some dark fibrils are present in the $\mathrm{H} \alpha$ filament channel, but due to seeing effects they are not sharp.

The EFC as observed by CDS shows a complex pattern of dark regions which appear nearly continuous around the neutral line, and which becomes more and more inhomogenuous away from it (see Fig. 2 (bottom)). In particular, our fine-tuned co-alignment proves that the elongated thick lateral extensions in EUV do not follow the $\mathrm{H} \alpha$ feet. Most of these extensions also have a left-bearing chirality, however we cannot infer a chirality rule for EFCs based on this single filament. Some isolated bright patches are 

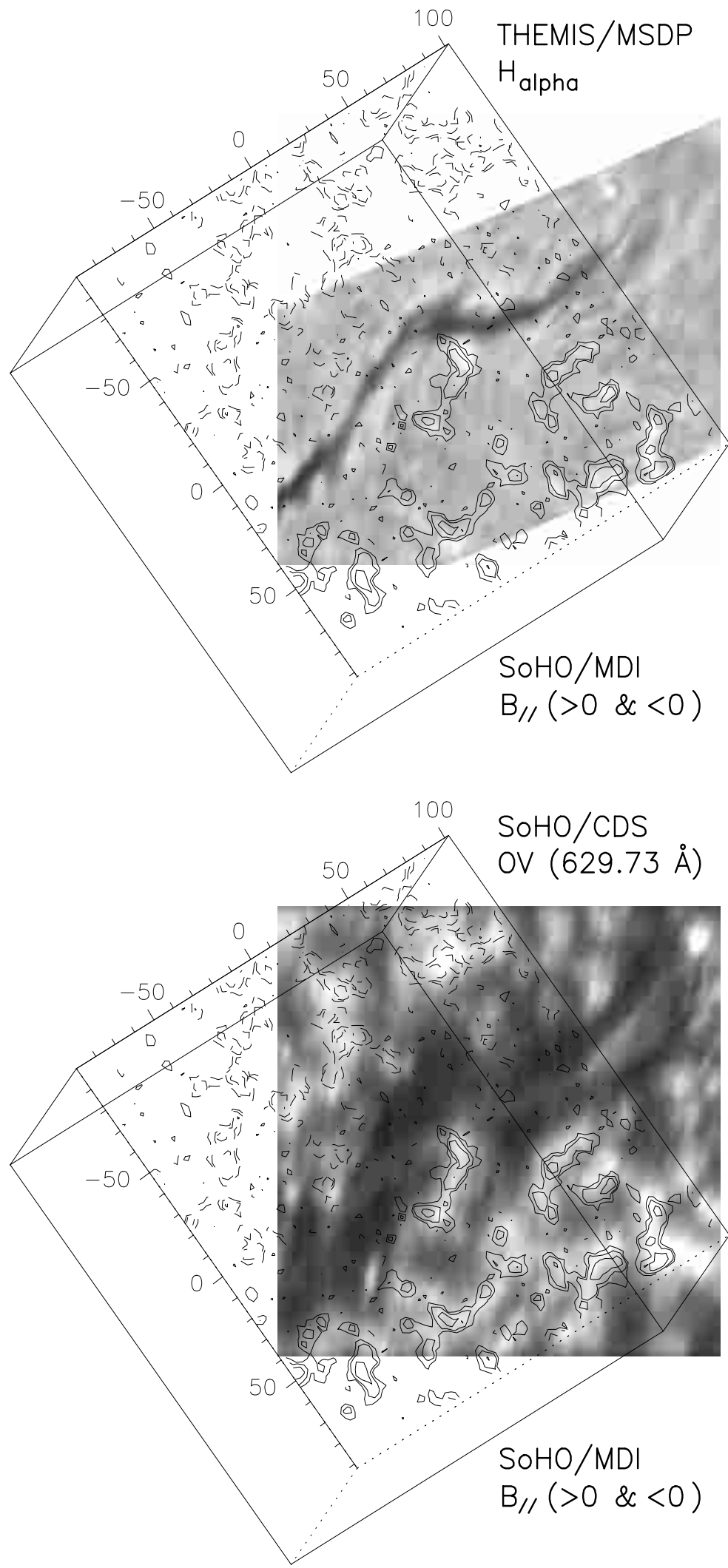

Fig. 2. Intensity maps of the filament channel observed on May 5, 2000 at E17 S21. Top: H $\alpha$ image obtained with THEMIS/MSDP at 07:52 UT. Bottom: Ov image (at $629.73 \AA$ ) obtained with SoHO/CDS at 08:12 UT. Both maps are overlaid with a SoHO/MDI magnetogram obtained at 8:00 UT. Isocontours of positive (resp. negative) longitudinal magnetic field are drawn in full (resp. dashed) lines. The thin edges correspond to the computational box of the model (see Fig. 3). Units are in $\mathrm{Mm}$. 
present in the outer parts of the EFC, which all correspond with magnetic field concentrations.

Interestingly, Figs. 1 and 2 show that the EFC has the same morphology as observed in Ov at $629.73 \AA$, in He II at $304 \AA$ and in Fe XII at $195 \AA$. With the continuum absorption model, this similarity suggests that EFCs observed with EIT (see e.g. Wang 2001) should also be due to the absorption of EUV lines, but in this case not only by the Lyman continuum of $\mathrm{H}(\lambda<912 \AA)$ but also by the continua of He I $(\lambda<504 \AA)$ and He II $(\lambda<227 \AA)$, as for arch filament systems observed in EUV (Mein et al. 2001).

Among the 6 wavelengths observed by CDS the Ov line is the only one that shows a thin elongated brightening in the middle of the EFC. This Ov spine is nearly continuous, and its location varies from the center to the edge of the $\mathrm{H} \alpha$ filament along its axis. We conjecture that this spine may be due to the emission from the upper prominence-corona transition region (PCTR). Its absence in the other observed wavelengths recorded by CDS is consistent with Chiuderi Drago et al. (1998, 2001), who reported that the emission of the PCTR at the top of filaments was negligible.

\section{Linear magnetohydrostatic models}

\subsection{Extrapolation of the magnetogram}

In Cartesian coordinates $\left(\boldsymbol{u}_{x}, \boldsymbol{u}_{y}, \boldsymbol{u}_{z}\right)$, where $z$ is the altitude, linear magnetohydrostatic (LMHS) fields can be calculated up to any height $z>0$ by solving the following equation derived by Low (1992):

$\boldsymbol{\nabla} \times \boldsymbol{B}=\alpha \boldsymbol{B}+f(z) \nabla B_{z} \times \boldsymbol{u}_{z}$,

where $\alpha$ is the force free parameter, using some given distribution $B_{z}^{*}(x, y)$ at $z=0$ as lower boundary conditions and with any function $f(z)$ which only varies with $z$. Following Low (1992) and Aulanier et al. (1998a), we chose:

$f(z)=a \exp \left(-\frac{z}{H}\right)$,

where $a$ is a measure of the pressure and gravity induced currents, and $H$ is the scale-height on which these currents decrease with height. In LMHS with periodic boundary conditions in $(x, y)$, Eq. (1) is solved under the following conditions:

$$
\lim _{z \rightarrow+\infty} B=0
$$

$\alpha, a, H=$ constant,

$\left.\alpha^{\prime}=\frac{\alpha}{\alpha_{\mathrm{c}}} \in\right]-1 ; 1[$

$\alpha_{\mathrm{c}}=\frac{2 \pi}{\max (D x, D y)}$

$a \in[0 ; 1]$,

where $(D x, D y)$ are the physical size of the computational box in $(x, y)$. So a compromize must be done between the choice of $\alpha$ and the size of the computational box.
For the purpose of this study, the lower boundary conditions are fixed by projecting the distribution of the line of sight component $B_{\|}$(obs) observed with SoHO/MDI onto the photospheric plane. So $B_{\|}(\operatorname{proj})(x, y)$ is obtained. As no existing data could provide the transverse components of the magnetic field, we assume that $B_{z}^{*}=$ $B_{\|}(\operatorname{proj}) / \cos \psi$, where $\psi$ is the angle between the line-ofsight $(l)$ and the vertical $(z)$ directions. For the filament located at E17 S21, $\psi=28.1^{\circ}$. The projection effects are accounted for by the use of the procedure described in Démoulin et al. (1997).

In previous filament models done with LMHS method (Aulanier et al. 1999, 2000) $B_{z}^{*}$ was modified in order to ensure the presence of a flux rope for high values of $\alpha$. Here we perform direct extrapolations of $B_{z}^{*}$ without any modification. On one hand, this choice leads to more difficulties in finding a good combination for the free parameters of the model, but on the other hand the obtained results should be more reliable than if the magnetogram was modified. Once $B_{z}^{*}$ is fixed, the remaining free parameters for the extrapolation are $D x, D y$ and $\alpha, a, H$.

\subsection{Computational box}

The orientation of the computational box is chosen so that its $y$ axis corresponds to the averaged direction of the observed $\mathrm{H} \alpha$ filament, which makes an $59^{\circ}$ angle with the solar North (see Figs. 1 and $2(t o p)$ ).

The choice for $D x$ is not easy because it represents the periodicity of the model perpendicularly to the filament axis. We can evaluate its value from the observations: Fig. 1 shows that the studied filament is located between two decaying active regions (AR 1 and 2) which form a succession of $[+,-,+,-]$ magnetic polarities. We approximate this observed property as a local quasi-periodicity in $x$. So for a distance in $y$ corresponding to the length of the central filament, we choose $D x$ to be the average distance between the two neutral lines of AR1 and AR2. We then obtain $192 \mathrm{Mm}$ as an approximated value for the quasi-periodicity. So we choose $D x=192 \mathrm{Mm}$.

Since $D x$ is chosen from observational constraints, it defines the maximum length scale of the box as well as the maximum allowed value for $\alpha$ in (see Eq. (6)). Therefore we have to choose $D y \leq D x$. However the length of the studied filament as observed in $\mathrm{H} \alpha$ is $\sim 245 \mathrm{Mm}$, so larger than $D x$ by $\sim 28 \%$. So in order to calculate a model for a filament portion as long as possible, we choose $D y=D x=192 \mathrm{Mm}$. The centering of the computational box is done so that the intersection of the $\mathrm{H} \alpha$ filament body with the box edges nearly occurs at the same position along $x$, so that the periodicity effects along $y$ are minimized. The positioning of the computational box above the observations is shown in Fig. 2.

We checked a posteriori the validity for the choice of the size $D=D y=D x$ of the computational box by recalculating several LMHS models with $150 \mathrm{Mm}<D<$ $400 \mathrm{Mm}$. We qualitatively found similar results as in the 
following for $180 \mathrm{Mm} \leq D \leq 230 \mathrm{Mm}$. Also in order to estimate the periodicty effects, we re-did a posteriori the calculations of this paper with different shifts $\delta y$ for the positioning of the box along the filament axis with $\delta y=$ $\pm D y / 2$. We noticed that the calculated magnetic dips (as described in Sects. 4.2, 4.3 and 5.3) are not very sensitive to shifts $\delta y= \pm D y / 4$. This shows that the periodicity effects along $y$ are not importamt in the LMHS models of this filament. More generally for weakly curved filaments, this small effect is not surprising: it can be explained by the strong alignment of the magnetic field along the $y$ axis for the high value for the force-free parameter $\alpha$ needed to produce a filament.

\subsection{Calculation of a grid of 35 models}

Our previous LMHS models of filaments (Aulanier et al. 1999, 2000) have shown that the parameter $a$ has to be chosen as high as possible so as to obtain significant departures from the force free state. So considering Eq. (7), we choose $a=1$. Since our objective is to produce a LMHS model that can be compared with the EUV observations, we only use the THEMIS/MSDP image to constrain the choice of the two remaining parameters $\alpha$ and $H$, independantly of the EUV data.

Following Aulanier \& Démoulin (1998), the left bearing orientation of the filament feet (see Sect. 2.3) implies positive values for the force free parameter $\alpha$. Five values for $\alpha^{\prime}$ (see Eq. (5)), are considered between 0.88 and 0.98 . This interval is chosen by considering previous analytical studies of the parameter space in which the conditions can be satisfied for a suitable filament model in a bipolar field, in linear force-free field invariant by translation (see Aulanier \& Démoulin 1998 (Fig. 2)). The minimum value is fixed below the threshold for which dips are present in $z>0$, and the maximum value is chosen so the top part of any resulting twisted flux tube does not reach unrealistically high altitudes in $z$ (typically $D / 2$ ), which is an artifact of linear force free field and LMHS models for high values of $\alpha^{\prime}$. Seven values for the scale-height $H$ of the pressure/gravity induced horizontal currents are chosen between 5 and $35 \mathrm{Mm}$, which correspond to half of the typical height of intermediate filaments as observed above the limb.

We then perform one LMHS extrapolation for each combination of $\alpha^{\prime}$ and $H$, with the boundary conditions defined in Sect. 3.2, with $512 \times 512$ mesh points in $x, y$. The magnetic field is calculated up to $z=D x / 2=96 \mathrm{Mm}$, which is above the typical height of intermediate filaments. Note that the LMHS method permits us to calculate the magnetic field up to any given height, provided that the solar curvature and solar wind effects can be neglected.

From each of these extrapolations, we calculate the resulting distribution of magnetic dips in the same way as described in Aulanier (2000), with spatial samplings of $\Delta x=\Delta y=3 \mathrm{Mm}$ and $\Delta z=1.5 \mathrm{Mm}$, and with a vertical filling of $300 \mathrm{~km}$ that corresponds to one pressure scale-height for typical prominence temperatures. In this way, we construct a grid of 35 models to be compared with the $\mathrm{H} \alpha$ data.

\section{Model of the $\mathrm{H} \alpha$ filament}

\subsection{Extraction of one model from the grid}

We overlay each model from the grid onto the THEMIS/MDSP data. We consider the shape of the body of the $\mathrm{H} \alpha$ filament as the feature to be matched by the $3 \mathrm{D}$ distribution of calculated dips. In the parameter space $\left(\alpha^{\prime}, H\right)$, the models which best fit the filament body are for $0.92 \leq \alpha^{\prime} \leq 0.96$ and $20 \mathrm{Mm} \leq H \leq 30 \mathrm{Mm}$.

In these intervals, the models with the lowest values of $\left(\alpha^{\prime}, H\right)$ reproduce the global shape of the filament body, but with many gaps (i.e. regions devoid of dips) between the filament feet. This is because there is neither enough field aligned currents to produce dips, nor enough effects of the plasma at high altitude to accentuate them. However for the largest values of $\left(\alpha^{\prime}, H\right)$ the gaps are filled by the distribution of dips, while at high altitude they form a structure that is much more aligned with the $y$ axis than the filament body is. This can be explained by the appearance of a twisted flux rope which reaches high enough altitudes at which the neutral line becomes almost aligned with $y$ since the high spatial frequencies present at $z=0$ have disappeared (Aulanier \& Démoulin 1998).

Thus, we choose the mean values in the interval, i.e. $\alpha^{\prime}=0.94$ (in physical units $\alpha=3.08 \times 10^{-8} \mathrm{~m}^{-1}$ ) and $H=25 \mathrm{Mm}$. With the spatial samplings given in Sect. 3.3 for the discretization in the calculation of dips, 5600 dips are found in the computational box.

Figure 3 (top) shows the $\mathrm{H} \alpha$ image of the filament overlaid with 3500 low altitude $(z<4 \mathrm{Mm})$ dips and 2100 high altitude $(z>4 \mathrm{Mm})$ dips, drawn up to $300 \mathrm{~km}$ from the bottom of the dip. The high altitude dips match most observed properties of the filament: the curved shape and the degree of continuity of its body, as well as the locations and orientations its lateral extensions.

\subsection{Curvature and continuity of the filament body}

We have noticed in Sect. 2.3 that the filament body had an "S-shape" with an elbow. The LMHS model shows that this shape is not due to "sigmoid" type field lines. The observed curvature is simply due to local deformations of the neutral line by strong magnetic concentrations, with the same polarity than the dominant one on their side of the neutral line. These polarities oppose to the tendency of the filament fields to be aligned along the neutral line. Note however that the presence of a larger scale sigmoid of the coronal loops around the filament (as in the models of Antiochos et al. 1994 and Titov \& Démoulin 1999) cannot be ruled out from our model, since the chosen size of our periodic computational box (see Sect. 3.2) does not permit to address this issue. 

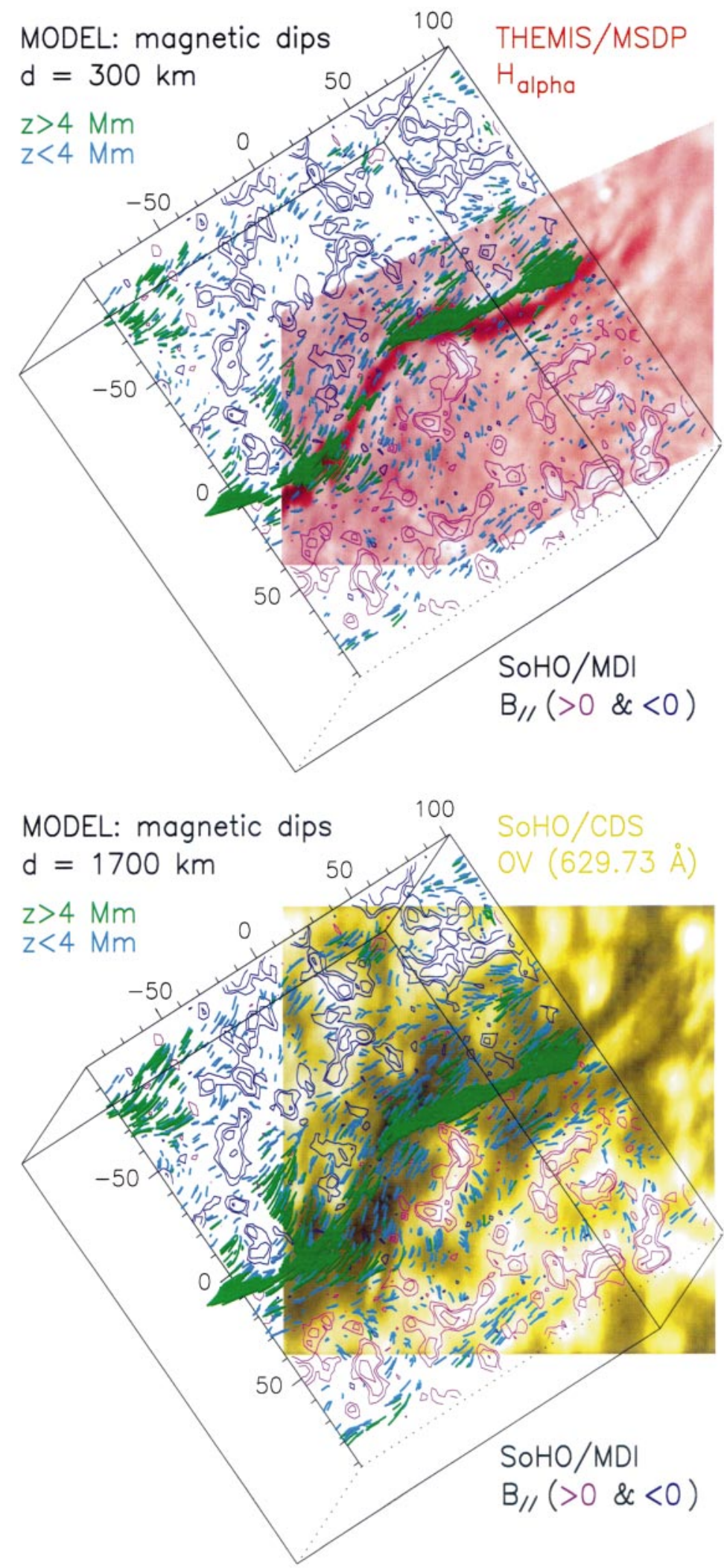

Fig. 3. Same as Fig. 2, overlaid by magnetic dips calculated from the LMHS model. 5600 dips are plotted with spatial samplings of $\Delta x=\Delta y=3 \mathrm{Mm}$ and $\Delta z=1.5 \mathrm{Mm}$. The high altitude dips (in green for $z>4 \mathrm{Mm}$ ) match the filament body and feet in $\mathrm{H} \alpha$. The low lying dips (in light blue for $z<4 \mathrm{Mm}$ ) match the wide EUV filament channel.

The observed continuity of the filament body along its axis (see Fig. $2(t o p)$ ) appears less straightforward to explain with the LMHS model, since Fig. 4 (bottom) shows that the distribution of locus of the dips in the filament 

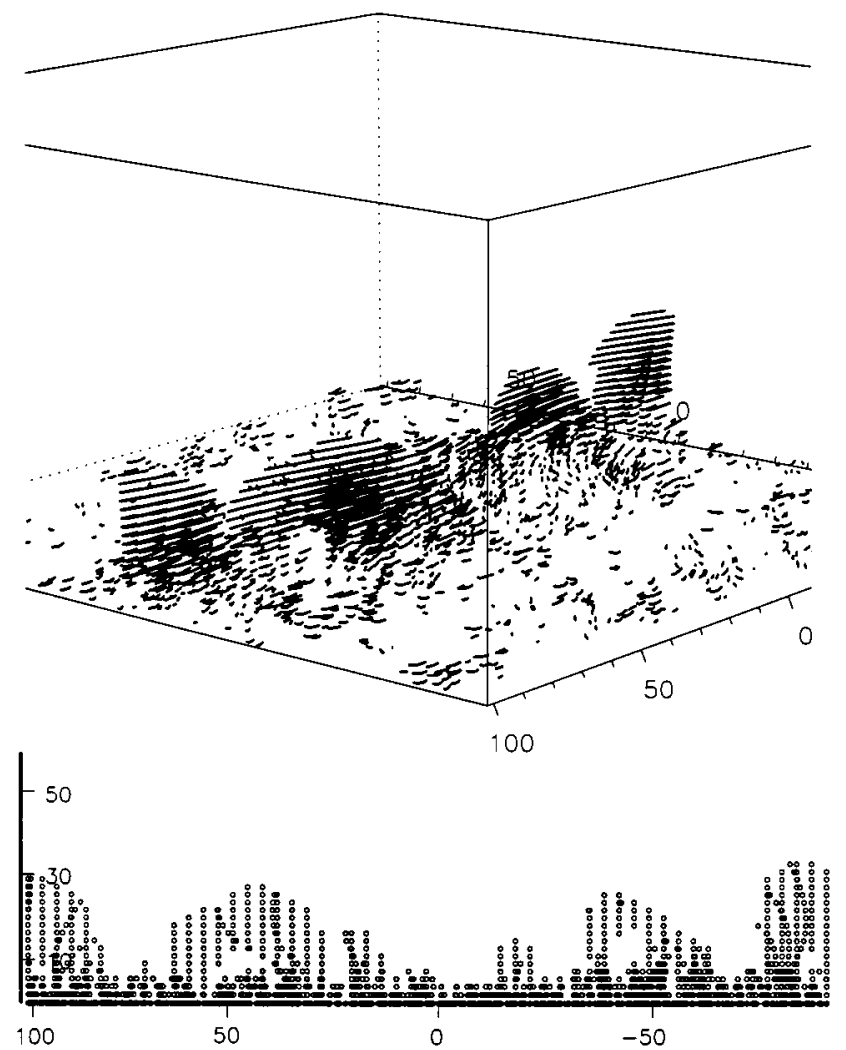

Fig. 4. Top: view in projection of the same 5600 magnetic dips as shown in Fig. 3. The dark lines are portions of dipped field lines drawn up to a height of $300 \mathrm{~km}$. Bottom: view from the side of the filament. Only the locus of the dips are marked with circles. Units are in Mm. The maximum altitude of the dips varies from 5 to $32 \mathrm{Mm}$ along the filament axis. The stripes on the top panel are due to the discrete sampling chosen for the drawing of magnetic dips (see Sect. 3.3) in which the field is nearly horizontal. This discretization, clearly apparent on the bottom panel, permits to emphasize the magnetic field direction and the projection effects on the top panel. A finer sampling only fills the thin elongated gaps between the dips: it replaces the stripes by a curtain-like black feature, which looks like real filaments/prominences viewed in projection.

body is far from being continuous, which is also confirmed by calculating dips with finer spatial samplings in $x, y$, $z$. In fact this discontinuous distribution of dips is not inconsistent with the quasi-continuity of the $\mathrm{H} \alpha$ filament. The vertical filling of dipped field lines up to $300 \mathrm{~km}$ also provides an horizontal extension for each dip, which can go up to $10 \mathrm{Mm}$. Also in most regions along the neutral line, isolated patches of dips are well aligned with the direction of the magnetic field. So the dipped field lines can fill most of the regions devoid of dips, leading to an apparent continuity of the filament body, as obtained in Fig. 3 (top). Figure $4(t o p)$ which is a view in projection of the same dips as shown in Fig. 3, confirms this interpretation. Also we have checked that this calculated distribution of dipped field lines matched fairly well the intensity iscocontours of the THEMIS/MSDP image in the filament.

\subsection{Location and magnetic field of filament feet}

Several lateral extensions to the filament body, knows as feet or barbs, are visible on the $\mathrm{H} \alpha$ data (see Figs. 1 and 2). This property is typical of $\mathrm{H} \alpha$ filaments, however as noted in Sect. 2.3, the feet of this filament are not related to any strong parasitic polarity. Nevertheless, the LMHS model clearly results in lateral dips which locations and orientations are consistent with the observed feet (see Fig. 3 $(t o p))$. Considering this result, we carefully examine the underlying magnetic field observed with SoHO/MDI.

Figure 5 shows that magnetic parasitic polarities do exist in the vicinity of feet (compare with Fig. 2), but they are at the limit of the instrument sensitivity: they have very weak field amplitudes of $B_{\|}=3-10 \mathrm{G}$. It is likely that these polarities are not due to the noise since many of them are extended over 1-10 pixels, even though their maximum value $(10 \mathrm{G})$ is always located on one single pixel (the pixel size of MDI is $1.977^{\prime \prime}$ ). This instrumental limitations may be responsible for the lower quality of the correlation between computed dips and observed feet as compared to the filament model of Aulanier et al. (1999).

Assuming that the MDI is reliable enough for such extended weak fields, we fully confirm our previous results on the interpretation of filament feet in terms of magnetic dips located underneath and next to the filament (Aulanier \& Démoulin 1998), their association with photospheric parasitic polarities (as observed by Martin 1990) and their location on the edge of these polarities (as observed by Aulanier et al. 1998b; Wang 2001).

\subsection{Magnetic twist}

We first emphasize that a large value for $H$ was necessary to produce a suitable filament model. This implies that plasma pressure and gravity may not be negligible for the structuring of filaments. However within the limitations of LMHS models, it is difficult to estimate if spatial variations of $\alpha$ in non-linear force-free models may replace or not the effects of plasma induced currents.

In the present work, a direct extrapolation of the observed magnetogram used as boundary conditions was done. This contrasts with Aulanier et al. (1999, 2000) were the magnetograms were modified at relatively large distances from the filament in order to ensure the presence a twisted flux rope at $z>0$ for high values of $\alpha$.

With this method, we find that the calculated topology for the filament magnetic field is more complex than a coherent flux rope. Figure 6 clearly shows many interruptions in the filament, manifested by some of the computed thick field lines which regularly reach the photosphere in non-parasitic strong polarities, i.e. which have the same polarity as the dominant one on the side of the neutral line where they are located. Despite the complex connectivity of the filament field lines at low altitude, at high altitude they show a global twist of $N \simeq 0.5$ turn in the computational box. Assuming that the twist per unit length does not vary within the whole $\mathrm{H} \alpha$ filament (which 


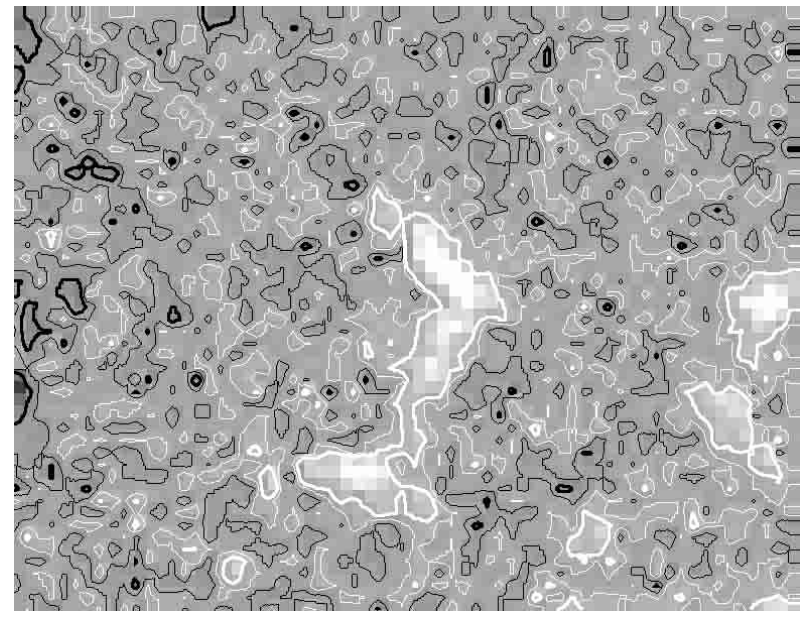

Fig. 5. Close-up of the SoHO/MDI magnetogram of $65 \times$ 50 pixels centered on the area where the $\mathrm{H} \alpha$ filament has its elbow and its northern foot (see Fig. 2 for comparison). The greyscale image shows individual pixels and the color table ranges in $\pm 100 \mathrm{G}$ for the line-of-sight magnetic fields $B_{\|}$. White (resp. black) interpolated contours are for $B_{\|}>0$ (resp. $<0$ ). Thick (resp. thin) contours are for $B_{\|}= \pm 10$ (resp. \pm 3 ) G. $B_{\|}>0$ (resp. $<0$ ) is the dominant polarity on the western/right (resp. eastern/left) side of the neutral line. The parasitic polarities are opposite in sign to the dominant polarity.

is about $245 \mathrm{Mm}$ long), the averaged twist of the portion of the flux tube where the filament material is visible is $N_{\mathrm{H} \alpha} \simeq 0.6$.

It is worth mentioning that the total twist of the full filament flux tube may be more than 0.6 for the following reasons. Firstly, the filament ends as observed in $\mathrm{H} \alpha$ may not correspond to the footpoints of the flux tube. This effect would increase the number of turns to $N_{\mathrm{H} \alpha}+\delta N$, with an increase which is likely to be $\delta N<0.5$. Secondly the EFC as observed by EIT is much longer than the studied $\mathrm{H} \alpha$ filament. Figure 1 (bottom) even shows that it may follow the switchabck which links EFC1 in the active region AR1. It is impossible to know if the full EFC corresponds to one single flux rope or not. If it does, assuming a constant twist per unit length and taking into account the length of EFC1 as well (which extends the EFC length to about $2 R_{\odot}$ ), we estimate an upper bound for the total twist of $N_{\max } \simeq 3.5+\delta N \simeq 4$.

In summary, we find that the length for one turn is $L \simeq 400 \mathrm{Mm}$, with an average flux tube radius projected onto the $(x, y)$ plane of $R \simeq 13 \mathrm{Mm}$ (see Fig. 6 (top right)). Assuming a circular cross-section of radius $R$ for the filament flux tube, the ratio between the toroidal field $B_{\phi}$ and the axial field $B_{\mathrm{r}}$ is then $B_{\phi} / B_{\mathrm{r}} \simeq 0.2$.

\section{The nature of the EUV filament channel}

\subsection{Location of plasma condensations}

Even though LMHS models do not address the mechanism of formation of plasma condensations in magnetic dips (see e.g. Poland \& Mariska 1986; Antiochos et al. 2000), they calculate higher densities in dips than in classical arcades, since the LMHS equation for the density (Low 1992):

$\rho=\rho_{0}(z)+\frac{1}{\mu g}\left(\frac{1}{2} \frac{\mathrm{d} f(z)}{\mathrm{d} z} B_{z}^{2}+f(z)(\boldsymbol{B} \cdot \nabla) B_{z}\right)$,

contains a curvature term of positive sign since $f(z) \geq 0$ (see Eqs. (2) and (7)). In Eq. (8), $\rho_{0}(z)$ is the hydrostatic density at at the altitude $z$ in the absence of magnetic field, $g$ is the amplitude of the gravitational acceleration and $\mu$ is the magnetic permeability. So the LMHS method predicts with good confidence that the plasma density in a dip is larger than in its surrounding.

However this method is an approximation, therefore it should not be considered to measure quantitative densities. For this reason, we need to estimate separately the amount of mass which is likely to be present in the dips, whose positions only are reliably calculated with the LMHS model. We have shown in the present and in previous filament models (Aulanier et al. 1999, 2000) that a vertical filling of magnetic dips of $300 \mathrm{~km}$ was consistent with the visibility of absorbing plasma as observed in $\mathrm{H} \alpha$. However in order to simulate observations in other absorbing wavelengths it is necessary to calculate different filling heights of magnetic dips to account for their different opacities as compared to $\mathrm{H} \alpha$.

\subsection{Estimate for the filling of magnetic dips}

In this section we discuss a simplified 2D model which permits, under several approximations, to estimate the vertical filling of magnetic dips as observed when a background EUV radiation is absorbed by the Lyman continuum of hydrogen (P. Heinzel, private communication).

We consider a $2 \mathrm{D}$ vertical distribution of identical dips in the $(x, z)$ plane, where $z$ is the altitude above the photosphere as well as the line-of-sight. We take $L_{z}$ for the vertical extension of this distribution of dips. Then we fill each of these dips by dense plasma, which can absorb background radiation emitted from below at the wavelength $\lambda$. We further assume density isocontours in the $(x, z)$ plane of the form $x=$ constant. In such a configuration, the optical thickness $\tau_{\lambda}$ for the wavelength $\lambda$ is given by:

$\tau_{\lambda}=\kappa_{\lambda}^{\prime} \rho L_{z}$

where $\rho$ is the plasma density and $\kappa_{\lambda}^{\prime}$ is the absorption coefficient normalized to standard plasma density.

Taking two different positions along a given dipped magnetic field line (or along the $x$ axis) corresponding to two plasma densities $\rho_{\lambda_{1}}$ and $\rho_{\lambda_{2}}$ chosen so as to obtain the same visibility (i.e. optical thickness) in both wavelengths $\lambda_{1}$ and $\lambda_{2}$, then Eq. (9) leads to:

$\kappa_{\lambda_{1}}^{\prime} \rho_{\lambda_{1}}=\kappa_{\lambda_{2}}^{\prime} \rho_{\lambda_{2}}$

Further assuming an isothermal hydrostatic equilibrium in each magnetic dip, so that $\rho_{\lambda_{i}}$ is the plasma density at 

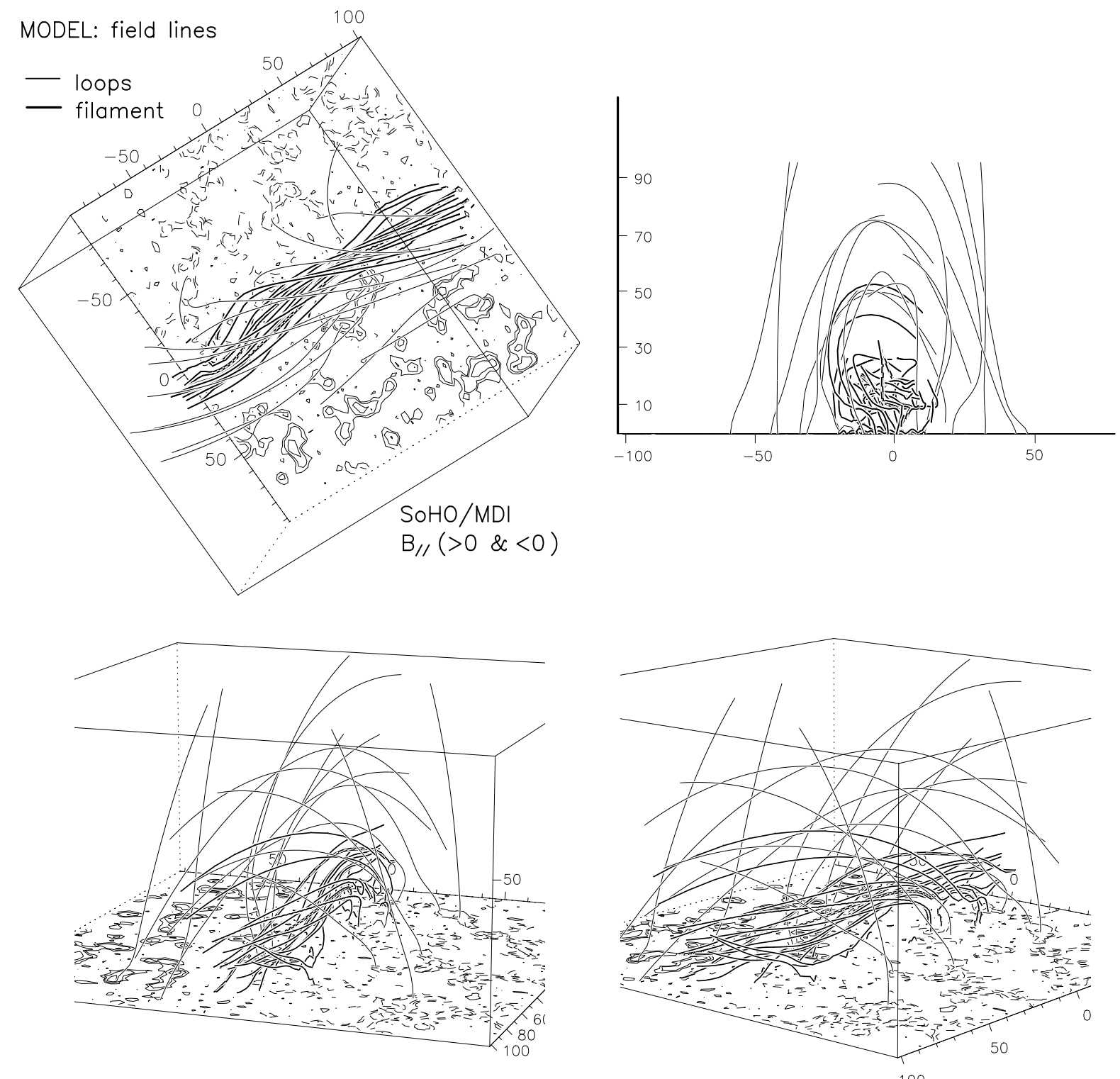

Fig. 6. Magnetic field lines computed from the same LMHS model as in Fig. 3. The field lines passing through the H $\alpha$ filament body and feet (resp. surrounding it) are drawn with thick (resp. thin) lines. The images show views in projection (top left) from earth, (top right) along the filament axis and (bottom) in projection. The bottom right projection is the same as for Fig. 4 (top).

the height $z_{\lambda_{i}}$ and taking $\Lambda$ for the pressure scale height, then Eq. (10) leads to:

$z_{\lambda_{2}}=z_{\lambda_{1}}+\Lambda \ln \left(\frac{\kappa_{\lambda_{2}}^{\prime}}{\kappa_{\lambda_{1}}^{\prime}}\right)$,

where $z_{\lambda_{i}}$ corresponds to the vertical filling for each dip as observed in the wavelength $\lambda_{i}$.

But for two different plasma densities (i.e. $\rho_{\lambda_{1}}$ and $\rho_{\lambda_{2}}$ ), the value for the ratio $\kappa_{\lambda_{2}}^{\prime} / \kappa_{\lambda_{1}}^{\prime}$ is difficult to estimate, because:

$\kappa_{\lambda}^{\prime}=\alpha_{\lambda} n_{i} / \rho$

where $\alpha_{\lambda}$ is the absorption cross-section for the given atomic transition and $n_{i}$ is the population of the atomic levels $i$ from which the absorption takes place, which depends on the temperature, the density and the radiation field. So the correct calculations of $\kappa_{\lambda}^{\prime}$ would require numerical NLTE models which are far beyond the scope of this paper. In order to give an approximated estimation for this ratio, we simplify the problem considering that $\kappa_{\lambda}^{\prime}$ does not depend on the plasma density $\rho$. This is qualitatively justified because the increasing of $\rho$ should also increase atomic level populations $n_{i}$.

With this simplification it is possible to evaluate $\kappa_{\lambda_{2}}^{\prime} / \kappa_{\lambda_{1}}^{\prime}$ for a given plasma density. Considering two wavelengths $\lambda_{1}$ and $\lambda_{2}$ and for one given position along $x$ in the vertical distribution of dips (so at fixed density $\rho$ ), then Eq. (9) leads to:

$\left.\frac{\kappa_{\lambda_{1}}^{\prime}}{\kappa_{\lambda_{2}}^{\prime}}\right|_{\rho}=\frac{\tau_{\lambda_{1}}}{\tau_{\lambda_{2}}}$ 
Then we take $\lambda_{1}$ for $\mathrm{H} \alpha$ (subscript $\alpha$ ) and $\lambda_{2}$ for the Lyman continuum of hydrogen (subscript c). We consider a typical prominence temperature $T \simeq 8000 \mathrm{~K}$ so that $\Lambda \simeq 300 \mathrm{~km}$. This is equal to $z_{\alpha}$, which we previoulsy estimated as the vertical filling of magnetic dips as observed in $\mathrm{H} \alpha$. So Eqs. (11) and (13) provide:

$$
\left(\frac{z_{\mathrm{c}}}{300 \mathrm{~km}}\right)=1+\ln \left(\frac{\tau_{\mathrm{c}}}{\tau_{\alpha}}\right),
$$

where $z_{\mathrm{c}}$ is the vertical filling of magnetic dips as observed in EUV lines absorbed in the Lyman continuum of hydrogen.

Since we now have an expression which only depends on the $\tau$ ratio, we can use the results from Heinzel et al. (2001), who computed with NLTE models an opacity ratio of $\tau_{\mathrm{c}} / \tau_{\alpha} \simeq 60-100$ for $T=8000 \mathrm{~K}$ and $\tau_{\alpha}=0.1-1$. In order to evaluate the maximum effect of the opacity for the observable filling of dips, we take the maximum value for $\tau_{\mathrm{c}} / \tau_{\alpha}$. So we estimate $z_{\mathrm{c}} \simeq 5.6 \Lambda \simeq 1700 \mathrm{~km}$.

Note that for a EUV line emitted at $\lambda<912 \AA$, a factor $\eta=(\lambda / 912)^{3}$ has to be multiplied to $\tau_{\mathrm{c}} / \tau_{\alpha}$ to obtain the opacity ratio between this line and $\mathrm{H} \alpha$ (see e.g. Chiuderi Drago et al. 2001; Heinzel et al. 2001). In the case of the Ov line observed by CDS (see Sect. 2.1), $\eta=0.33$. Taking this modification into account, Eq. (14) leads to $z(\mathrm{OV}) \simeq 0.8 z_{\mathrm{c}}$. In the present $2 \mathrm{D}$ isothermal and hydrostatic model, the effect of $\eta$ is small due its logarithmic expression. So in the following we neglect this effect because the precise estimation of the value for the vertical filling of dips is likely to be more sensitive to the other approximations listed above.

\subsection{EUV absorption observed in magnetic dips}

It is only at this stage that we overlay the CDS image in Ov $(629.73 \AA)$ with the magnetic dips of the LMHS model of the $\mathrm{H} \alpha$ filament, as calculated in Sect. 4.1 but now computed up to height of $1700 \mathrm{~km}$ as estimated in Sect. 5.2. This overlay is shown in Fig. 3 (bottom). Despite the limitations of the LMHS model and the strong approximations for the estimation of the filling of dips, a striking good match is found between the dark EUV regions and the projected distribution of dips onto the plane of the observations. Figure 3 (bottom) shows that a nearly one to one correspondance is obtained.

Apart for the central filament itself, which is nearly undistinguishable from the rest of the EFC, most of the dark regions located aside of the filament correspond to the locus of low lying magnetic dips, located above the photosphere and below $4 \mathrm{Mm}$ in altitude. The horizontal extension of these dips can at some places reach 10$20 \mathrm{Mm}$, which permits the filling of most of the dark regions, parallely to the local magnetic field direction. Among all the reproduced dark regions, note in particular the long and thick South-Western extension from the filament, the inhomogeneous structure located along the filament on its South-Eastern side only and several "ring-shaped" regions connected to the Northern part of the filament.

This suggests that wide EFCs have the same nature as the $\mathrm{H} \alpha$ filament: they are composed of suspended $(z \geq 0)$ magnetic dips in which plasma condensations are present. If the typical temperatures in these condensations are those of prominences so that neutral hydrogen can be present, the good match between the LMHS dips and the EUV data supports the absorption interpretation (see Sect. 1). Moreover, the distribution of dips in the EFC far from the filament are weakly extended in altitude. This is opposite to the filament $\mathrm{H} \alpha$ body and feet in which more dips are integrated along the line of sight. Since these low altitude dips are not correlated with any obvious $\mathrm{H} \alpha$ feature, the LMHS model is qualitatively consistent with opacity ratios calculated by Heinzel et al. (2001).

\subsection{Topology of the magnetic field}

We have discussed in Sect. 3.3 how the periodicity effects along $y$ only weakly affect the calculated magnetic dips. However it is obvious that this periodicity does not permit to calculate the connectivity of long field lines which come out of the computational box. Despite of this limitation, the LMHS model clearly shows that the dips of the EFC are formed within the lower parts of long sheared arcades that overlay the filament flux tube (see Fig. 6). So the dips in the extended EFC are not located in field lines which belong to the filament flux tube: its radius projected onto the $(x, y)$ plane is too small to cover the full width of the EFC.

As for the filament feet observed in $\mathrm{H} \alpha$ (see Sect. 4.3), every dip in the EFC is associated with weak photospheric parasitic polarities located between stronger magnetic polarities of the network. In particular, the South-Western elongated left-bearing extension of the EFC which is very well matched by magnetic dips (as described in Sect. 5.3 and shown in Fig. 3 (bottom)), is clearly associated with extended and dispersed weak negative polarities (as shown on the right portion of Fig. 5).

The local topologies of the magnetic field around each of these polarities are all the same: field lines tangential to the photosphere are present on one edge of each polarity, so forming "bald patches" (as defined by Titov et al. 1993). Direct representation of the computed topology is difficult since it involves a combination of large and small scales, so we refer the reader to Bungey et al. (1996) and to Aulanier et al. (1998b (Figs. 2a and 6a)) where this topology was calculated for theoretical models.

\subsection{Broadening of EFCs by wide EUV feet}

The chirality and the magnetic topology (see Sects. 2.3 and 5.4) of the laterally extended regions in the studied EFC are exactly the same as what we found for filament feet observed in $\mathrm{H} \alpha$ (Aulanier et al. 1999, 2000). Therefore, in order to homogeneize the terminology of 


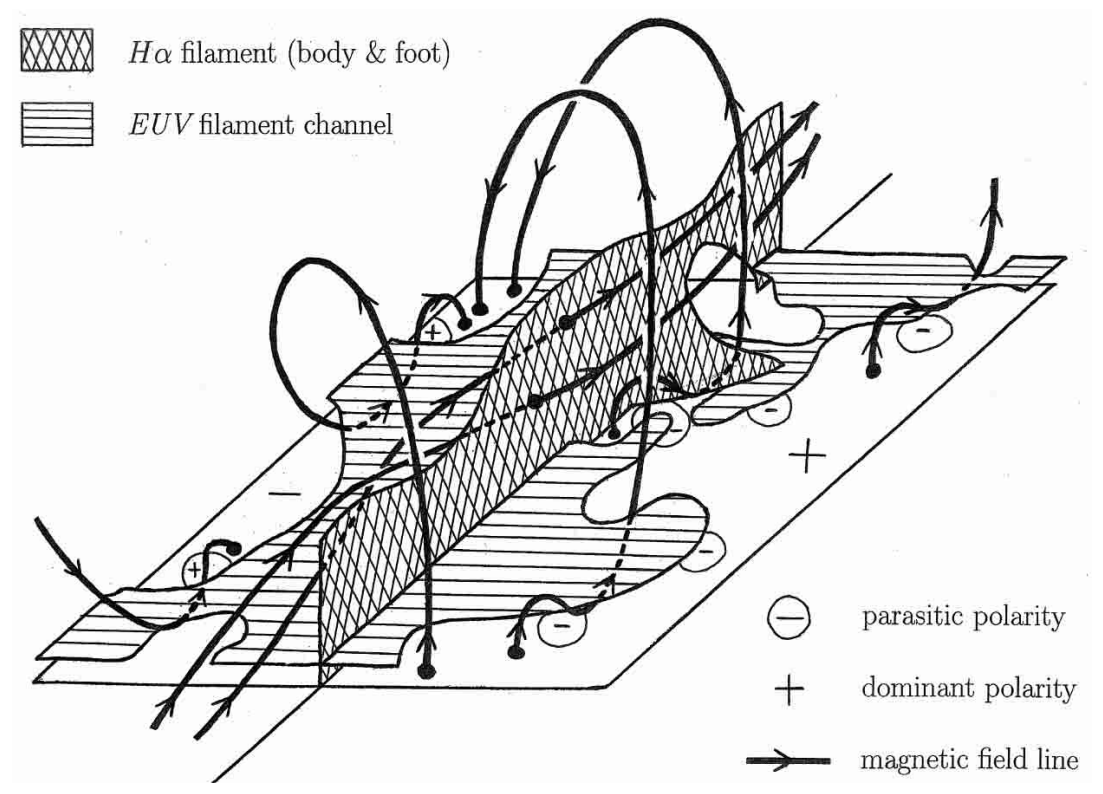

Fig. 7. Summary of the LMHS model. It shows the relation between the topology of the magnetic field and the location of the plasma condensations in and around the filament, which absorb the $\mathrm{H} \alpha$ and EUV (for $\lambda<912 \AA$ ) background radiation.

physically similar solar phenomena as observed in different wavelengths, we suggest to redefine the wide extended regions of EUV filament channels as the "EUV feet" of their central filament.

Even if the large filling of each dip plays a nonnegligible role in the broadening of EFCs, the contribution of EUV feet is obviously dominant. For example, the longest extended foot described in Sect. 5.4 is about $100 \mathrm{Mm}$ long and its maximum width near the neutral line is about $50 \mathrm{Mm}$. Also, the EUV broadening of the Eastern part of the $\mathrm{H} \alpha$ filament occurs on its Southern side only. Both these examples, as well as the observed "ring shaped" dark regions, are clearly incompatible with the single contribution from the large filling of dips. The frequently observed degree of inhomogeneity near the boundaries of EFCs (see Sect. 2.3) becomes readily interpreted by the inhomogeneous distribution of parasitic polarities in decaying active regions, which is consistent with the SoHO/MDI magnetogram (see Fig. 5).

It follows that any EFC related to an active region filament should be narrower and more homogeneous than those related to intermediate and quiescent filaments. Also EFCs in any active region should progressively broaden and become inhomogeneous, as the active region emerges and decays during several solar rotations. This is fully consistent with observations of the long term evolution of active regions (van Driel-Gesztelyi 1998 (Figs. 1 and 2)). We would also like to mention that our results on this studied EFC are also fully consistent with the recent He II (at $304 \AA$ ) and magnetic observations of Wang (2001) of several filaments, despite Wang's claims (in his Sect. 7) and his cartoon interpretation (in his Fig. 8).

A schematic which summarizes our results of our interpretation of $\mathrm{H} \alpha$ and EUV observations is shown in Fig. 7. We emphasize that this schematic is not a cartoon.
It displays the results of the calculated 3D LMHS model in simplified way, so that large and small scales can be visualized in one single frame and so that each feature is clearly identifiable.

\section{Discussion}

\subsection{Comparison with other models and observations}

The topology of the magnetic field as calculated with the 3D LMHS model was discussed in Sect. 4.4. We have shown that in spite of several partial interruptions by photospheric magnetic concentrations that have the same polarity as the dominant one on the side of the neutral line where they are located, the filament flux tube is twisted by less than one turn for the length of the filament as observed in $\mathrm{H} \alpha$.

On one hand, the lower portions of the magnetic field lines rooted in these non parasitic polarities are not associated with any observed $\mathrm{H} \alpha$ filament feet. On the other hand, no field line from the filament flux tube reach the $z=0$ plane inside parasitic polarities. In great majority, the field lines rooted in parasitic polarities are connected to the same side of the neutral line by small arcades, while a few are connected to the other side of the neutral line by flat arcades underlying the filament flux tube. So our LMHS model is opposite to the conceptual interpretations by Martin et al. (1994) and Wang (2001), which propose that filament bodies and feet are formed in classical undipped arcades.

Assuming that the filament flux tube is not much longer than the $\mathrm{H} \alpha$ filament (about $245 \mathrm{Mm}$ ), its moderate amount of twist $(N \sim 0.6-1)$ makes the LMHS model consistent both with weakly twisted flux rope models (e.g. van Ballegooijen 1999; Amari et al. 1999 (Fig. 2); 
Titov \& Démoulin 1999) and with differentially sheared arcade models (Antiochos et al. 1994; DeVore \& Antiochos 2000). But if the flux tube links the studied filament with F1 in AR1 (see Fig. 1), then its length (about $2 R_{\odot}$ ) and number of turns $(N \sim 3.5-4)$ would make it more consistent with the flux rope models of filaments in switchbacks (van Ballegooijen et al. 2000; MacKay et al. 2000). But whatever is the total length of the flux tube, its ratio $B_{\phi} / B_{\mathrm{r}} \simeq 0.2$ makes the LMHS model inconsistent with flux ropes in which the poloidal and the axial field magnitudes are comparable (see e.g. Amari et al. 1999 (Fig. 3); Gibson \& Low 2001).

Finally, we wish to emphasize that views in projection, both of the dips only (Fig. $4(t o p)$ ) and of the full field lines (Fig. 6 (bottom)) do not permit us to identify the calculated weak twist, which is consistent with most $\mathrm{H} \alpha$ observations of filaments. Also these projections show that the magnetic dips become more and more shallow as the altitude increases in the filament. Under the present limitations of observations of flows in filaments, using Doppler measurements and/or high cadence filtergram observations, our numerically calculated magnetic field is also consistent with $\mathrm{H} \alpha$ and EUV observations of dynamic condensations as observed on the disc or at the limb (see e.g. Malherbe et al. 1983; Schmieder et al. 1991; Zirker et al. 1998; Wang 1999).

Our calculated LMHS model predicts that, apart from the top of the prominence body where the magnetic field is nearly flat, any given dynamic condensation cannot move all along the prominence body and feet due to the total depth of their dips (J. T. Karpen, private communication). To the authors' knowledge, this remains to be tested by observations.

\subsection{Visibility of condensations in EFCs}

The $\mathrm{H} \alpha$ filament structure is undistinguishable in the EUV observations. But the consistency found between the hydrostatic filling of magnetic dips (Sect. 5.2) and the observed EUV feet (Sect. 5.3) suggests that the calculated filling of dips may also hold in the filament body. Consequently, plasma condensations in filaments may also be as long a $10-20 \mathrm{Mm}$. This is a challenge for coupled radiative and thermodynamic models of plasma condensations in magnetic dips (Poland \& Mariska 1986; Antiochos et al. 2000; Heinzel \& Anzer 2001). While these elongated condensations are not completely observed in $\mathrm{H} \alpha$ for filaments on the disc (due to the opacity of $\mathrm{H} \alpha$, see Heinzel et al. 2001), they may be observed in other wavelengths (not only in EUV) and/or above the limb in prominences. Under such different observable conditions and following Sect. 4.2, these elongated dips may fill some gaps that are frequently observed in filament bodies in $\mathrm{H} \alpha$. This may play a role in interpreting observations of prominences at the limb which show different patterns as observed in different wavelengths (see e.g. Kucera et al. 1998; Wang et al. 1998).
Our co-aligned observations (see Fig. 2) reveal that EUV feet are not always related to $\mathrm{H} \alpha$ feet. From the LMHS modeling, this can be explained and generalized to every EFC. It is obvious that any given $\mathrm{H} \alpha$ foot should always be located inside a EUV foot, in particular close to the filament body. Also, the absence of a EUV foot at some given location naturally implies the absence of any $\mathrm{H} \alpha$ foot at the same place. But far from the filament, long $\mathrm{H} \alpha$ feet (or dark fibrils) will only be observable co-spatially with EUV feet if the size and flux of the related parasitic polarities is strong enough and if the arcades overlaying the filament are sufficiently inclined toward the photosphere, so that the vertical distribution of low lying dips responsible for the EUV absorption will be thick enough (along the line of sight) to sufficiently absorb $\mathrm{H} \alpha$ radiation so that an observable contrast with the chromospheric background can be obtained.

\subsection{Existence of EFCs without filaments}

We calculated several linear force free models $(a=H=0)$ in the same box as defined in Sect. 3.2, and we compared the distribution of dips with the EUV images (see Fig. 8). We found that for increasing values of $\alpha$ from 0 (potential field) to $\alpha_{\mathrm{c}}$, the orientation of the calculated dipped field line progressively tends to align with the neutral line direction, and also that their vertical extent slightly increases.

In the absence of pressure and gravity effects, we find that no dip can be matched with the $\mathrm{H} \alpha$ filament for $\alpha^{\prime}<0.98$, because in this case the magnetic shear is not sufficient to create the weakly twisted flux tube above the neutral line responsible for high altitude dips. Even with $0 \leq \alpha^{\prime}<0.98$, some portions of the complex structure of the EUV dark regions can still be matched. This is natural because $\alpha$ has a weak effect on the small parasitic polarities responsible for the low lying dips, because they correspond to high spatial frequencies in $x$ and $y$ (Aulanier \& Démoulin 1998). But the edges of the EFC are poorly reproduced, and the distribution of low lying dips is too dispersed to match the observed degree of homogeneity of the EFC. This dispersion is mainly caused by the unability of dipped field lines to fill the gaps between separated patches of dips, because for $\alpha^{\prime}<0.98$ the conditions given in Sect. 4.2 are not satisfied.

So we predict that dark EFCs with wide EUV feet can be observed, even when no filament is present. This holds not only when prominence material is unobservable in some given wavelength, but also when there is no magnetic configuration capable of supporting any prominence condensation at high altitudes.

This is consistent with the existence of $\mathrm{H} \alpha$ filament channels without filaments (see e.g. Martin 1990), but for different physical reasons: around a given neutral line, the presence of an $\mathrm{H} \alpha$ filament channel simply indicates a large amount of magnetic shear, whereas the presence of an EFC mostly indicates the presence of low lying magnetic dips, for which the magnetic shear is not as 

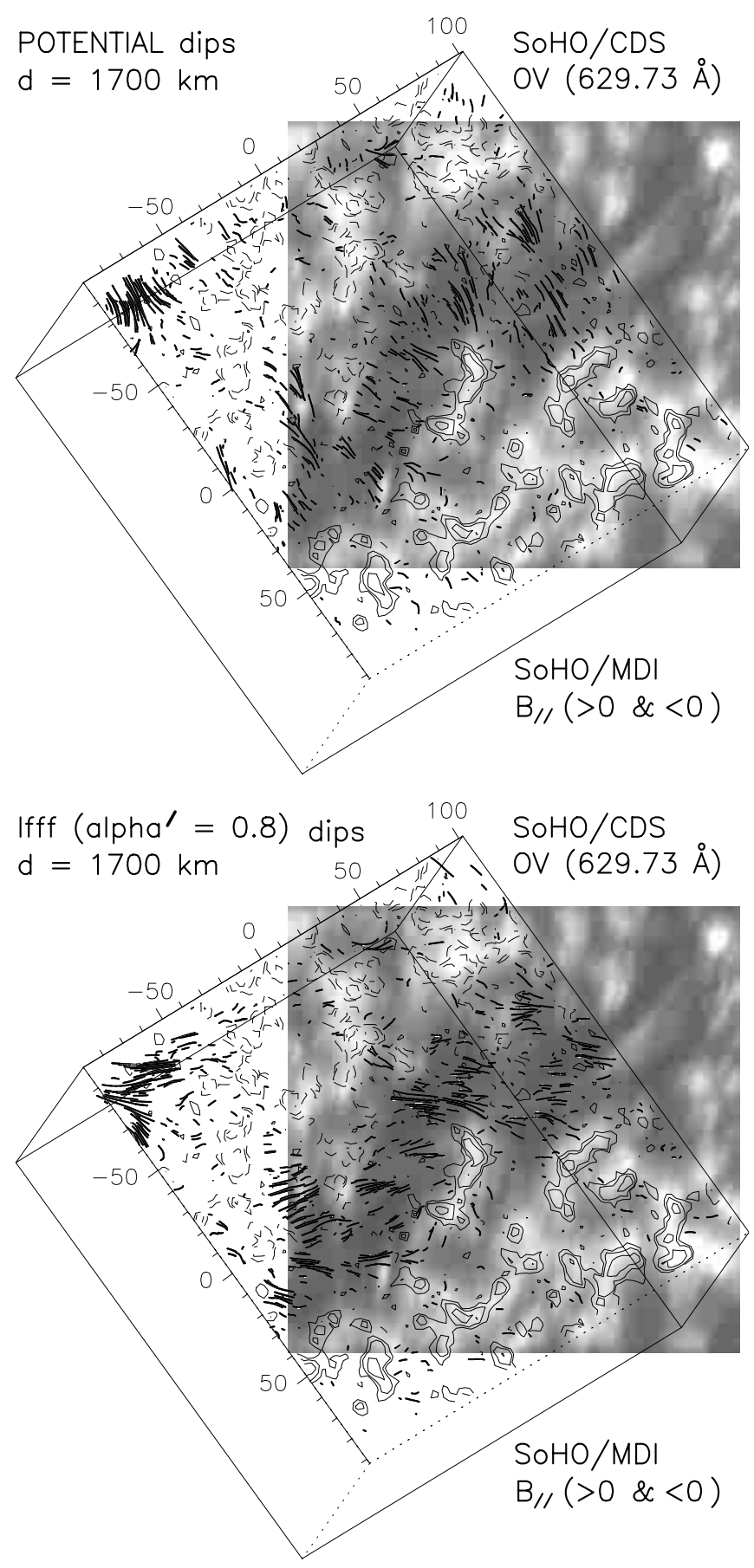

Fig. 8. Same as Fig. 3 (bottom) but here the dips are calculated from $(t o p)$ a potential field extrapolation $(\alpha=a=H=0)$ and (bottom) a linear force free field $(a=H=0)$ extrapolation with $\alpha^{\prime}=0.8$. All the dips are low in altitude $(0 \leq z \leq 6 \mathrm{Mm})$. To some degree, the dips match some wide EUV absorbing features, but not the $\mathrm{H} \alpha$ filament.

important as the distribution of dispersed weak photospheric parasitic polarities.

Considering low spatial resolution observations at different EUV wavelengths, the absence of a filament may be difficult to detect in a given EFC, because of typically high opacities not only in the Lyman continuum of hydrogen, but also for other wavelengths in which filaments are typically observed (e.g. Ly $\alpha$ ): with high opacities, no difference can be observed between thin layers of low lying dips or thick layers such as filaments. However, if isolated fine structures exist within EFCs, high spatial resolution observations may permit to deduce the presence of a filament both through the degree of homogeneity of the EFC and through the orientation of the related fine structures.

\subsection{Loading low-lying dips by cool material}

Since the magnetic dips which form the lateral extensions of filaments are very shallow (about 10-20 Mm long for $1700 \mathrm{~km}$ high) and are formed at low altitude $(z \leq 4 \mathrm{Mm}$ for EUV feet, and about twice as much for $\mathrm{H} \alpha$ feet), plasma injection into these dips may be very easily achieved through simple MHD effects which may not involve complex thermal processes as required for the formation of prominence condensations at much higher altitudes (see e.g. Poland \& Mariska 1986; Antiochos et al. 2000).

One possibility is shear Alfvèn waves driven by photospheric motions. They can easily lift up chromospheric material along the magnetic field, which will eventually get trapped in the suspended dips. Another possibility arises from the bald patch topology that we computed. Magnetic reconnection in the bald patch separatrix can provide the direct filling of higher and higher dips by chromospheric material as reconnection proceeds. Note that this process differs from the reconnection scenario of Wang (1999, 2001), which aims at producing dynamic condensations in undipped field lines.

It should be mentioned that the possibility of magnetic reconnection in bald patches was debated by Karpen et al. (1991) due to the thickness of the photospheric layer, but counter-arguments were provided by theory (Low 1991; Billinghurst et al. 1993) and by models of observed flares (Aulanier et al. 1998a) and fibrils (Mandrini et al. 1999). Note that in the case of filament channels, the reconnection scenario in bald patches is also supported by Kucera et al. (1999), who reported isolated bright EUV features located on the side of a filament and observed in Si IV, which were correlated with calculated flux tubes rooted in bald patches, and which were interpreted as magnetic loops heated by reconnection and filled by chromospheric evaporation.

Even though a bald patch reconnection model appears consistent with some observations, it will have to be tested with new observations and models. In particular the issue of heating of the chromospheric plasma will have to be addressed, since EUV absorption by the Lyman continuum of Hydrogen requires cool temperatures $\left(T \leq 10^{4} \mathrm{~K}\right)$.

\subsection{Upper prominence-corona transition region}

In the Ov line at $629.73 \AA$ only, a peculiar bright elongated continuous spine is observed in the central part of the EFC (see Fig. 2 (bottom)). We conjectured in Sect. 2.3 
that this spine could be interpreted as the upper prominence corona transition region (PCTR) of the filament. We examine below this possibility.

Careful examination of the overlay of the $\mathrm{H} \alpha$ image with the modeled dips (see Fig. $2($ top $)$ ) reveals that at some places the uppermost dips in the filament body are shifted from the center of the $\mathrm{H} \alpha$ filament. We have confirmed that theses shifts were due to projection effects in the complex thin volume which forms the filament body. Also, Fig. 2 (bottom) shows that the same uppermost dips are well co-aligned with the bright Ov spine. So it is very likely that this spine is the result of EUV emission from the uppermost dips, which cannot be absorbed at higher altitudes since there are no higher dips. So the LMHS model is consistent with an upper PCTR interpretation for this Ov spine located on top of the filament.

The distribution of dips in 3D (see Fig. 4) shows that the altitude of the uppermost dips strongly varies nonmonotonically between 5 and $32 \mathrm{Mm}$ along the filament body. This suggests that the observed continuity of the Ov spine is an artifact caused by projection effects. With the LMHS model, the spine is consistent with the emission of several discrete upper PCTRs which are associated with different magnetic dips at various altitudes, which belong to different magnetic field lines. This result may have strong implications for multi-dimensional radiative transfer modeling of prominences.

\section{Role of EFCs in the mass loading of CMEs}

During the filament eruption, the field lines forming its main flux tube (described in Sect. 4.4 and shown by thick lines in Fig. 6) will expand dynamically. Regardless of the EUV feet, the large vertical filling of hydrostatic dips by $1700 \mathrm{~km}$, which is about 5.6 pressure scale heights, implies that the filament body could be about $50 \%$ more massive than what can be measured in $\mathrm{H} \alpha$. This extra mass, which can be observed in EUV as opposed to $\mathrm{H} \alpha$, will naturally be loaded into the associated coronal mass ejection (CME). For the specific class of "three-part CMEs" that incorporate a leading front followed by a cavity which surrounds a bright core, this extra mass should be confined on the edges of the bright core, since the latter corresponds to the erupting filament (see the review of Low 2001).

The relative number of calculated low/high altitude magnetic dips (see Sect. 4.1) suggests that there can be at least the same amount of mass in EUV feet than in their related central filament, but located at low altitudes, in thin volumes on top of which the filament stands (see Figs. 4 and 7). A fully time-dependant MHD simulation of the eruption of the studied filament (or any other) would be required to study quantitatively the role of its EUV feet in the mass loading of the CME. This is far beyond the scope of this paper, however it is still possible to infer the evolution of the EFC as its filament erupts through simple argumentations.

The filament flux tube expansion described above will push up, or aside, the overlaying sheared arcades (shown by thin lines in Fig. 6), as typically calculated by 3D MHD simulations of erupting flux ropes (see e.g. Amari et al. 2000 (Fig. 3)). The top parts of the arcades that will be pushed up will eventually be injected into the CME all together with the filament flux tube. The bottom parts of these expanding arcades, in which the low-lying dips which form the EFC are located (see Sect. 5.4), whether they are pushed aside or injected into the CME, are likely to be affected by the eruption in the following way: each cool condensation of the EFC will remain at low heights if its pre-eruptive dip is maintained, or will fall down to the chromosphere along the field if its pre-eruptive dip is straightened. These two possibilities arise from the competition between the tendancy of field lines to become quasi-vertical due the field opening and the flux of the parasitic polarities that tends to keep the field lines locally bent toward the photosphere at low altitude. After the expanding phase, the open field lines will close back through reconnection, loosing a fraction of their magnetic shear, i.e. lowering the value of $\alpha$ in the linear force free field approximation.

This likely scenario, which still needs to be confirmed by time-dependant simulations, predicts that the mass of the wide low-lying EFC, i.e. of the EUV feet, will not contribute to the CME when the filament erupts. It also predicts that an EFC can be partly or nearly fully maintained after its filament eruption, since even a strong decrease of $\alpha$ does not remove all the low lying dips that are responsible for the EUV absorption in the EFC (see Sect. 6.3).

\section{Summary}

Wide EUV filament channels have been frequently interpreted by the Hydrogen Lyman continuum absorption of EUV lines shortward of $912 \AA$ in cool massive condensations (Orrall \& Schmahl 1979, 1979; Chiuderi Drago et al. 1998, 2001). Heinzel et al. (2001) and Schmieder et al. (2002) have also shown that these EUV channels are much more extended as compared to their main $\mathrm{H} \alpha$ filament. The object of this work was to propose, through a case study, a general physical model for EUV filament channels, and to address their role in the mass loading of coronal mass ejections (CMEs), which is an important issue for estimating their kinetic energies.

We calculated in 3D the magnetic field in and around one observed filament, with the linear magnetohydrostatic (LMHS) extrapolation method (Low 1992; Aulanier et al. 1998a). The model was built from a SoHO/MDI magnetogram so as to match the $\mathrm{H} \alpha$ observations from THEMIS/MSDP, independantly of the EUV data from SoHO/CDS. We fully confirmed our previous results on the magnetic structure of filament $\mathrm{H} \alpha$ feet in terms of magnetic dips (Aulanier \& Démoulin 1998; Aulanier et al. $1999,2000)$. We also found that the observed continuity of the filament $\mathrm{H} \alpha$ body was ensured by the filling of gaps between isolated patches of dips by their horizontal extension due to their vertical filling by plasma up to $300 \mathrm{~km}$. 
We found a complex topology for the filament flux tube, in which a global twist of about 0.6 turn could be identified.

Then we compared the LMHS model with the observed EUV filament channel (EFC). A striking one to one correspondance was found between the EFC and the distribution of every calculated magnetic dip. The joint analysis of the model and the data proved the presence of plasma condensations which can potentially absorb the background EUV radiation. Also this analysis revealed their location, magnetic nature and likely evolution when the filament erupts. The results, which have been sketched in Fig. 7, can be summarized and generalized as follows:

The absorbing condensations in EFCs are formed where the magnetic field is dipped, in the lower $\simeq 1700 \mathrm{~km}$ portions of their field lines. In the central parts of EFCs, the dips are the same than those forming the filament body and feet as observed in $\mathrm{H} \alpha$. In the wide lateral extensions of EFCs, the dips are formed above dispersed photospheric parasitic polarities and are grouped in thin layers at low altitude $(0 \leq z \leq 4 \mathrm{Mm}$, where $z=0$ is the altitude of the MDI magnetogram). As for $\mathrm{H} \alpha$ features, the vertical filling of dips provides the observed degree of continuity in EFCs. So the physical properties of EUV extensions are very similar to those of $\mathrm{H} \alpha$ feet, but both are not necessarily co-spatial. In order to homogeneize the terminology for solar phenomena that have the same physical nature, we redefined the wide extensions of EFCs by "EUV feet". The 3D distribution of dips is qualitatively consistent with the respective visibility of these structures in $\mathrm{H} \alpha$ and in EUV as derived from the opacity ratios calculated by Heinzel et al. (2001) from the NLTE radiative transfer models.

These findings on the magnetic nature of EFCs and the calculated coronal magnetic field led us to discuss several implications. We proposed a possible scenario for plasma injection in the low-lying dips of EUV and $\mathrm{H} \alpha$ feet through magnetic reconnection in bald patches. We have shown that EFCs could exist in the absence of the high altitude magnetic configuration capable of supporting cool material, so in the absence of filaments. We have also shown that the apparently continuous Ov bright spine, located at the center of the EFC, could be interpreted as the emission from several isolated upper prominence-corona transition regions (PCTRs). We have shown that each upper PCTR was located ontop of the uppermost filament magnetic dips, at various altitudes and in different magnetic field lines, depending on their position along the filament axis.

During a filament eruption, some of the mass of the filament body is known to be injected into the CME. The ratio for the filling of the dips by $\operatorname{cool}(T \simeq 8000 \mathrm{~K})$ hydrostatic material as observed in $\mathrm{H} \alpha$ and in EUV suggests that this ejected mass should be $\simeq 1.5$ times larger than what is estimated from $\mathrm{H} \alpha$ observations of filaments. Since this mass is located within the outer regions of each dip of the filament flux tube, its location in the propagating CME should remain on the edge of the main erupting prominence. But the topology of the coronal magnetic field in and around EFCs implies that the large amount of mass contained in their wide low-lying EUV feet will not be loaded into their associated CMEs. We calculated that after a CME, the loss of magnetic shear in the corona will not remove all the low lying dips, even if the potential state is reached. So wide EFCs should be partly maintained, even right after their filament has erupted and before it eventually reforms.

We have shown that the morphology of EFCs is the same when observed in several transition region and coronal lines whose wavelengths are shortward of $912 \AA$ as recorded by $\mathrm{SoHO} / \mathrm{CDS}$, but also in lower wavelengths such as $304 \AA$ (He II) and $195 \AA$ (Fe XII). It naturally follows that our interpretation of filament channels observed in these wavelengths should also be valid. In this case, the absorption by the continua of He I $(\lambda<504 \AA)$ and He II $(\lambda<227 \AA)$ must be taken into account, as shown by Mein et al. (2001) for emerging arch filament systems. Observations in these EUV wavelengths are typically done by SoHO/EIT and by TRACE. Also they will be provided by STEREO/SECCHI/EUVI with the unprecedented capability of doing stereoscopic observations. So in terms of morphology, these instruments will be well suited to test further our interpretation and our predictions. However EUV spectroscopy will be needed to obtain more quantitative estimates of the mass in filaments and in their channels.

Acknowledgements. We thank P. Heinzel and K. Tziotziou for their critical comments and their help in the writing of Sect. 5.2. We also thank J.-M. Malherbe and P. Mein for their assistance with the THEMIS observations, as well as P. Démoulin, L. van Driel-Gesztelyi, J. T. Karpen, J.-C. Vial and Y.-M. Wang for stimulating discussions. We are grateful to the MEDOC and the THEMIS staff for having conducted the coordinated observing campaign from which the CDS and the MSDP data used in this paper were obtained. We also thank the EIT and MDI consortiums as well as the French solar database BASS 2000 for providing the other data used in this paper. The THEMIS telescope is operated by INSU/CNRS (France) and CNR (Italy) on the island of Tenerife in the Observatorio del Teide of the IAC (Spain). SoHO is a project of international cooperation between ESA and NASA. The work of G.A. was funded by the Centre National d'Etudes Spatiales (CNES).

\section{References}

Amari, T., Luciani, J.-F., Mikić, Z., \& Linker, J. 1999, ApJ, 518, L57

Amari, T., Luciani, J.-F., Mikić, Z., \& Linker, J. 2000, ApJ, 529, L49

Antiochos, S. K., Dahlburg, R. B., \& Klimchuck, J. A. 1994, ApJ, 420, L41

Antiochos, S. K., MacNeice, P. J., \& Spicer, D. S. 2000, ApJ, 536, 494 
Aulanier, G., \& Démoulin, P. 1998, A\&A, 329, 1125

Aulanier, G., Démoulin, P., Schmieder, B., Fang, C., \& Tang, Y. H. 1998a, Sol. Phys., 183, 369

Aulanier, G., Démoulin, P., van Driel-Gesztelyi, L., Mein, P., \& DeForest, C. 1998b, A\&A, 335, 309

Aulanier, G., Démoulin, P., Mein, N., et al. 1999, A\&A, 432, 867

Aulanier, G., Srivastava, N., \& Martin, S. F. 2000, ApJ, 543, 447

Billinghurst, M. N., Craig, I. J. D., \& Sneyd, A. D. 1993, A\&A, 279,589

Bungey, T. N., Titov, V. S., \& Priest, E. R. 1996, A\&A, 308, 233

Chae, J. 2000, ApJ, 540, L115

Chiuderi Drago, F., Bocchialini, K., Lamartinie, S., et al. 1998, in IAU 167 Colloq., Aussois, ed. D. Webb, B. Schmieder, \& D. Rust, ASP Conf. Ser., 150, 55

Chiuderi Drago, F., Alissandrakis, C. E., Bastian, T., Bocchialini, K., \& Harrison, R. A. 2001, Sol. Phys., 199, 115

d'Azambuja, L., \& d'Azambuja, M. 1948, Annales de l'Observatoire de Paris, 6, 7

Delannée, C. 2000, JASTP, 62, 1471

Delannée, C., Delaboudinière, J.-P., \& Lamy, P. 2000, A\&A, 355,725

Démoulin, P., Bagalá, L. G., Mandrini, C. H., Hénoux, J.-C., \& Rovira, M. G. 1997, A\&A, 325, 305

DeVore, C. R., \& Antiochos, S. K. 2000, ApJ, 539, 954

Forbes, T. G. 2000, JGR, 105, A10, 23153

Gosling, J. T., Hildner, E., MacQueen, R. M., et al. 1974, JGR, 79,4581

Gibson, S. E., \& Low, B. C. 2001, JGR, 105, A8, 18187

Heinzel, P., \& Anzer, U. 2001, A\&A, 375, 1082

Heinzel, P., Schmieder, B., \& Tziotziou, K. 2001, ApJ, 561, L223

Howard, R. A., Sheeley, N. R. Jr., Michels, D., \& Koomen, M. J. 1985, JGR, 90, A9, 8173

Hundhausen, A. J. 1988, in Proc. of the sixth international solar wind conference, TN306+Proc, ed. V. Pizzo, T. E. Holzer, \& D. G. Sime, NCAR, Boulder, 181

Karpen, J. T., Antiochos, S. K., \& DeVore, C. R. 1991, ApJ, 382,327

Klimchuk, J. A. 2001, in Space Weather, ed. P. Song, H. J. Singer, \& G. Siscoe (Washington: American Geophys. Union), Geophys. Monograph, 125, 143

Kucera, T. A., Andretta, V., \& Poland, A. I. 1998, Sol. Phys., 183,107

Kucera, T. A., Aulanier, G., Schmieder, B., Mein, N., \& Vial, J.-C. 1999, Sol. Phys., 186, 259

Low, B. C. 1991, ApJ, 381, 295

Low, B. C. 1992, ApJ, 399, 300

Low, B. C. 2001, JGR, 106, A11, 25141
MacKay, D. H., Gaizauskas, V., \& van Ballegooijen, A. A. 2000, ApJ, 544, 1122

Malherbe, J.-M., Schmieder, B., Ribes, E., \& Mein, P. 1983, A\&A, 119, 197

Mandrini, C. H., Deng, Y. Y., Schmieder, B., et al. 1999, in 3rd Advances in Solar Physics Euroconference, ed. B. Schmieder, A. Hofmann, \& J. Staude, ASP Conf. Ser., 184, 276

Marqué, C., Lantos, P., \& Delaboudinière, J.-P. 2002, A\&A, submitted

Martin, S. F. 1990, IAU Colloq. 117, ed. E. Tandberg-Hanssen, Lect. Notes Phys. 363 (Springer-Verlag), 1

Martin, S. F., Bilimoria, R., \& Tracadas, P. W. 1994, in Solar Surface Magnetism (Kluwer Ac. Pub.), 303

Mein, N., Schmieder, B., DeLuca, E. E., et al. 2001, ApJ, 556, 438

Orrall, E. Q., \& Schmahl, E. J. 1976, Sol. Phys., 50, 365

Orrall, E. Q., \& Schmahl, E. J. 1979, ApJ, 231, L41

Poland, A. I., \& Mariska, J. T. 1986, Sol. Phys., 104, 303

Schmieder, B. 1989, in Dynamics and structure of quiescent solar prominences, ed. E. R. Priest (Kluwer Academic Publishers, Dordrecht, Holland), 15

Schmieder, B., Raadu, M. A., \& Wiik, J. E. 1991, A\&A, 252, 353

Schmieder, B., Delannée, C., Yong Deng Yuan, Vial J.-C., \& Madjarska, M. 2000, A\&A, 358, 728

Schmieder, B., Tziotziou, K., \& Heinzel, P. 2002, submitted

Subramanian, P., \& Dere, K. P. 2001, ApJ, 561, 372

Tang, F. 1987, Sol. Phys., 107, 233

Titov, V. S., Priest, E. R., \& Démoulin, P. 1993, A\&A, 276, 564

Titov, V. S., \& Démoulin, P. 1999, A\&A, 351, 707

van Ballegooijen, A. A. 1999, Magnetic helicity in space and laboratory plasma, ed. M. R. Brown, R. C. Canfield, \& A. A. Pevstov, Geophys. Monograph, 111, 213

van Ballegooijen, A. A., Priest, E. R., \& MacKay, D. H. 2000, ApJ, 539, 983

van Driel-Gesztelyi, L., Schmieder, B., Aulanier, G., et al. 1998, in IAU 167 Colloq., Aussois, ed. D. Webb, B. Schmieder, \& D. Rust, ASP Conf. Ser., 150, 366

van Driel-Gesztelyi, L. 1998, in 2nd Advances in Solar Physics Euroconference, ed. C. E. Alissandrakis, \& B. Schmieder, ASP Conf. Ser., 155, 202

Vial, J. C. 1990, IAU Colloq. 117, ed. E. Tandberg-Hanssen, Lect. Notes Phys. 363 (Springer-Verlag), 106

Wang, H., Chae, J., Gurman, J., \& Kucera, T. 1998, Sol. Phys., 183, 91

Wang, Y.-M. 1999, ApJ, 520, L71

Wang, Y.-M. 2001, ApJ, 560, 456

Zirker, J. B., Martin, S. F., \& Engvold, O. 1998, Nature, 396, 440 